\title{
Synthetic seismograms for finite sources in spherically symmetric Earth using normal-mode summation
}

\author{
Tianshi Liu $\cdot$ Haiming Zhang
}

Received: 14 February 2017/ Accepted: 19 June 2017/Published online: 28 August 2017

(C) The Author(s) 2017. This article is an open access publication

\begin{abstract}
Normal-mode summation is the most rapidly used method in calculating synthetic seismograms. However, normal-mode summation is mostly applied to point sources. For earthquakes triggered by faults extending for as long as several $100 \mathrm{~km}$, the seismic waves are usually simulated by point source summation. In this paper, we attempt to follow a different route, i.e., directly calculate the excitation of each mode, and use normal-mode summation to obtain the seismogram. Furthermore, we assume the finite source to be a "line source" and numerically calculate the transverse component of synthetic seismograms for vertical strike-slip faults. Finally, we analyze the features in the Love waves excited by finite faults.
\end{abstract}

Keywords Normal-mode summation · Synthetic seismogram $\cdot$ Finite fault $\cdot$ Surface waves

\section{Introduction}

The calculation of synthetic seismograms is one of the most important topics in seismology, because on the one hand, synthetic seismogram is the bridge that connects the theory of seismology and the observational data, and on the other hand, it is crucial to structure inversion and rupture process inversion. Roughly speaking there are three types of methods to calculate synthetic seismograms. The first type is numerical method, e.g., the finite difference method

T. Liu $\cdot$ H. Zhang $(\bowtie)$

Department of Geophysics, School of Earth and Space Sciences, Peking University, Beijing 100871, People's Republic of China e-mail: zhanghm@pku.edu.cn

T. Liu

e-mail: dash2007@163.com
(Boore 1972), the finite element method (Bielak et al. 2003) and the spectral element method (Komatitsch and Tromp 1999, 2002). Numerical methods are usually quite flexible. They are available for very complex structures. But the computational costs are usually high, and the compromise between accuracy and efficiency is often inevitable. The second type is asymptotic method, e.g., the generalized ray method (Gilbert and Helmberger 1972) and the WKBJ method (Chapman 1978). The asymptotic methods typically have high efficiency, but only effective for computing the high-frequency component. The third type is semi-analytical method, e.g., the discrete wavenumber method (Bouchon and Aki 1977) and the R/T coefficient method (Luco and Apsel 1983; Kennett and Kerry 1979) for stratified half-space, and the normal-mode summation method (Dahlen and Tromp 1998) for spherically symmetric Earth model.

Normal-mode summation is one of the most widely used methods in simulating teleseismic waves. After the 1960 Chile earthquake, seismologists did a great amount of studies on normal modes and gradually developed the normal-mode summation method. Gilbert (1971) explicitly showed the normal-mode summation representation of the displacement in elastic media. Singh and Ben-Menahem (1969a, b) calculated the excitation of each mode by a point source in spherically symmetric Earth. Takeuchi and Saito (1972) derived the equations that govern the normal modes. Tanimoto (1984) obtained the formulae to calculate long-period synthetic seismograms using normal-mode summation. Woodhouse (1988) developed the numerical method to calculate the radial eigenfunction, which made it possible to calculate synthetic seismograms using normalmode summation. Dahlen and Tromp (1998) integrated the previous work and constructed a comprehensive and 
compact framework for theoretical global seismology, which includes the normal-mode theory, normal-mode summation method and other related topics.

However, normal-mode summation is mostly used for point sources. In order to calculate the synthetic seismogram for a finite source, point source summation is usually used (Bouchon 1980a, b; Song and Helmberger 1996): the fault is discretized into small sub-faults which can be treated as point sources; the seismogram for each sub-fault is calculated using normal-mode summation and then the seismogram for the finite fault is obtained by adding the seismograms of the sub-faults together. Analytical or semianalytical methods are sometimes applied to calculating the synthetic seismograms of finite faults (Ben-Menahem and Singh 1987; Israel and Kovach 1977; Saikia and Helmberger 1997; Stump and Johnson 1982).

In this work, we expand the normal-mode summation method to the finite fault case. We first derive the excitation of each mode and then add them together to obtain the seismogram. We assume that the fault is a "line source": it expand transversely but concentrate at a certain depth radially, which take into account the effect of propagation in the transverse direction, but ignore that of the propagation in the radial direction. We represent the normal mode in the form of generalized spherical harmonics (Phinney and Burridge 1973; Yang et al. 2010). Next, we use the radial eigenfunction of MINEOS (Woodhouse 1988) and calculate the numerically results of the transverse component in the case of the vertical strike-slip fault as example. We then observe some features in the Love wave of the finite source, and we use an intuitive model to explain these features based on Chap.10 of Aki and Richards (2002).

\section{Normal-mode summation for finite source}

\subsection{From point source to finite source}

Suppose that the displacement at $\boldsymbol{x}$ excited by a point source at $\boldsymbol{x}^{\prime}$ can be written in the form of normal-mode summation as

$\boldsymbol{g}\left(\boldsymbol{x}, t ; \boldsymbol{x}^{\prime}\right)=\sum_{k} A_{k}\left(\boldsymbol{x}^{\prime}, t\right) \boldsymbol{s}_{k}(\boldsymbol{x})$,

in which $s_{k}$ is the eigenfunction with index $k$, and $A_{k}$ represents the excitation of mode with index $k$ by the point source at $\boldsymbol{x}^{\prime}$. Note that here the source does not have to be a body force, so $\boldsymbol{g}$ is not necessarily the Green's function. For a finite source with a spatial and temporal amplitude distribution $D\left(x^{\prime}, \tau\right)$, the displacement at $\boldsymbol{x}$ is

$$
\begin{aligned}
\boldsymbol{u}(\boldsymbol{x}, t) & =\int_{\Sigma_{f}} \int_{0}^{T_{f}} \boldsymbol{g}\left(\boldsymbol{x}, t-\tau ; \boldsymbol{x}^{\prime}\right) D\left(\boldsymbol{x}^{\prime}, \tau\right) \mathrm{d} \tau \mathrm{d} S \\
& =\sum_{k}\left(\int_{\Sigma_{f}} \int_{0}^{T_{f}} A_{k}\left(\boldsymbol{x}^{\prime}, t-\tau\right) D\left(\boldsymbol{x}^{\prime}, \tau\right) \mathrm{d} \tau \mathrm{d} S\right) \boldsymbol{s}_{k}(\boldsymbol{x}),
\end{aligned}
$$

where $\Sigma_{f}$ is the fault plane, $T_{f}$ is the total rupture time. Thus, the seismogram of the finite fault can also be written in the normal-mode summation form

$\boldsymbol{u}(\boldsymbol{x}, t)=\sum_{k} \mathscr{A}_{k}(t) \boldsymbol{s}_{k}(\boldsymbol{x})$,

where the excitation of mode with index $k$ is

$\mathscr{A}_{k}(t)=\int_{\Sigma_{f}} \int_{0}^{T_{f}} A_{k}\left(\boldsymbol{x}^{\prime}, t-\tau\right) D\left(\boldsymbol{x}^{\prime}, \tau\right) \mathrm{d} \tau \mathrm{d} S$.

The excitation of mode with index $k$ by a double-couple $\boldsymbol{M}$ is well known (Dahlen and Tromp 1998; Yang et al. 2010),

$A_{k}\left(\boldsymbol{x}^{\prime}, t\right)=\left(\boldsymbol{M}: \varepsilon_{k}^{*}\left(\boldsymbol{x}^{\prime}\right)\right) \frac{1-\mathrm{e}^{-\sigma_{k} t} \cos \omega_{k} t}{\omega_{k}^{2}}$.

Substitute Eq. (5) in Eq. (4), and let

$\dot{\boldsymbol{m}}\left(\boldsymbol{x}^{\prime}, \tau\right)=\boldsymbol{M} D\left(\boldsymbol{x}^{\prime}, \tau\right)$

we obtain

$$
\begin{aligned}
\mathscr{A}_{k}(t)= & \int_{\Sigma_{f}} \int_{0}^{T_{f}}\left(\dot{\boldsymbol{m}}\left(\boldsymbol{x}^{\prime}, \tau\right): \boldsymbol{\varepsilon}_{k}^{*}\left(\boldsymbol{x}^{\prime}\right)\right) \\
& \times \frac{1-\mathrm{e}^{-\sigma_{k}(t-\tau)} \cos \omega_{k}(t-\tau)}{\omega_{k}^{2}} \mathrm{~d} \tau \mathrm{d} S .
\end{aligned}
$$

\subsection{GSH representation for spherically symmetric} Earth

Any tensor can be decomposed in generalized spherical coordinate, and the components can be represented using generalized spherical harmonics (GSH). We assume that the Earth structure is spherically uniform, and we can write the normal modes using GSH. Then, we use Eqs. (7) and (2) to obtain the final solution of the displacement excited by finite source.

The normal mode of the spherical symmetric Earth is (Dahlen and Tromp 1998)

$\boldsymbol{s}_{k}(r, \theta, \phi)=\sum_{\alpha=0, \pm 1} s_{n l}^{\alpha}(r) Y_{l m}^{\alpha}(\theta, \phi) \hat{\boldsymbol{e}}_{\alpha}$,

where $Y_{l m}^{\alpha}$ is the generalized scalar harmonics, $\hat{\boldsymbol{e}}_{\alpha}$ is the base of generalized spherical coordinate. For a spheroidal or a toroidal mode, the index $k$ is the same as $n, l, m$. 
$s_{n l}^{ \pm 1}=\frac{1}{\sqrt{2}} V_{n l}, \quad s_{n l}^{0}=U_{n l}$

for spheroidal mode, and

$s_{n l}^{ \pm 1}= \pm \frac{1}{\sqrt{2}} W_{n l}, \quad s_{n l}^{0}=0$

for toroidal mode, where $U_{n l}, V_{n l}$ and $W_{n l}$ are radial eigenfunctions.

Substituting Eq. (8) into Eqs. (7) and (2), we can derive the representation of seismogram using GSH. But before this, we make some simplifications. First, without loss of generality, we can put our spherical coordinate such that the line connecting the pole and the origin is perpendicular to the fault plane (see Fig. 1). In such coordinate, the coordinate of the receiver is $\{r, \theta, \phi\}$, the coordinate of some point on the fault plane is $\left\{r^{\prime}, \theta^{\prime}, \varphi\right\}$. We set up a local coordinate $\{\hat{\boldsymbol{\xi}}, \hat{\boldsymbol{\phi}}, \hat{\boldsymbol{v}}\}$ on the fault plane. Using this coordinate system,

$\mathrm{d} S=r^{\prime} \sin \theta^{\prime} \mathrm{d} \xi \mathrm{d} \varphi$.

Next, we assume that the fault only expands transversely, but concentrates at a certain depth (line source). Unlike the point source approximation, which concentrates the seismic moment at a single point, the line source approximation assumes that the seismic moment concentrates on a line. It is a more general assumption than the point source approximation, and it captures the

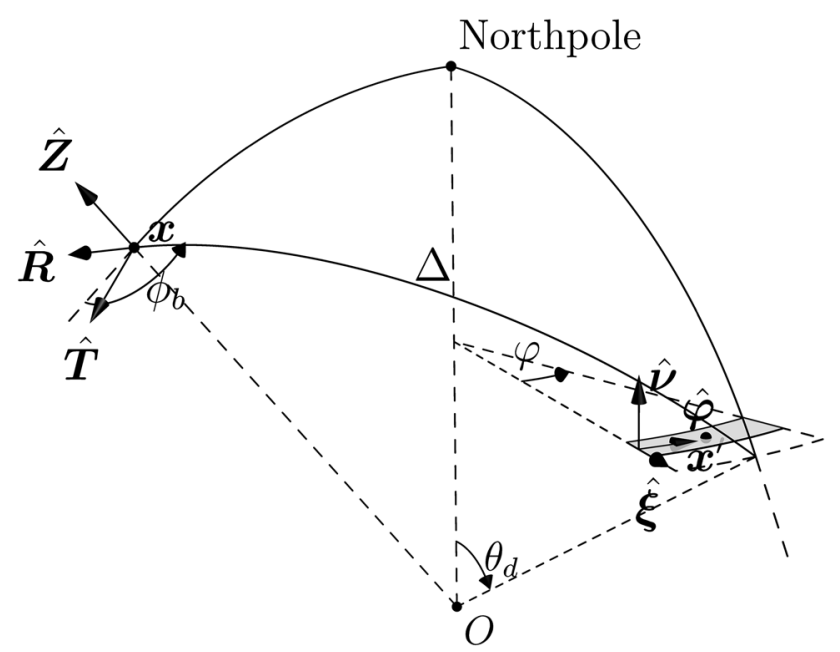

Fig. 1 Geometry of the receiver and the finite source. The global spherical coordinate is selected such that the line connecting the origin and the "Northpole" is perpendicular to the fault plane. The local coordinate at the receiver $(\boldsymbol{x})$ is the "ZRT" coordinate. The basis of local coordinate at the receiver is $\{\hat{\boldsymbol{\xi}}, \hat{\boldsymbol{\phi}}, \hat{\boldsymbol{v}}\}$. The reference point of the source is $\boldsymbol{x}^{\prime}$, which can be an arbitrary point near the fault (e.g., epicenter). The angular distance between the reference point and the receiver is defined as the epicentral distance $\Delta$. The back-azimuth is $\phi_{b}$ effect of the lateral propagation of the rupture, but it is still far from enough to capture the full finite fault feature of the earthquake. The line source approximation is valid because the impact of the transverse propagation of rupture is far more significant compared with the radial propagation. Thus, the integral on the fault can be simplified as

$\mathrm{d} S=r_{f} w \sin \theta_{d} \mathrm{~d} \varphi$,

where $w$ is the width of the fault, $\theta_{d}$ is the dip-angle of the fault plane, and $r_{f}$ is the distance between the fault and the origin.

Using the aforementioned spherical coordinate and the line source approximation, we obtain the GSH representation of displacement triggered by the finite fault. We use ${ }_{\gamma}^{K} u^{q}$ to represent the displacement in $q$ direction ( $q=Z, R, T$ for each direction of the ZRT coordinate), related to $K$ type mode ( $K=S, T$ for spheroidal and toroidal mode) triggered by $\gamma$ component of $\operatorname{sip}(\gamma=\varphi, \xi$ for strike-slip and dip-slip component). For simplicity, we show only the result of displacement that related to toroidal mode and triggered by strike-slip component

$$
\begin{aligned}
& { }_{\varphi}^{T} u_{n l}^{Z}=0 \text {, } \\
& { }_{\varphi}^{T} u_{n l}^{R}=\sum_{n, l}-2 \sin \theta_{d} W_{n l}(r)^{T} \mathscr{S}_{n l}^{2} \mathfrak{R}\left\{\mathrm{e}^{\mathrm{i} \phi_{b}} \frac{{ }_{\varphi}^{T} \mathscr{K}_{n l}^{-2,+1}+{ }_{\varphi}^{T} \mathscr{K}_{n l}^{+2,+1}}{2}\right\}
\end{aligned}
$$

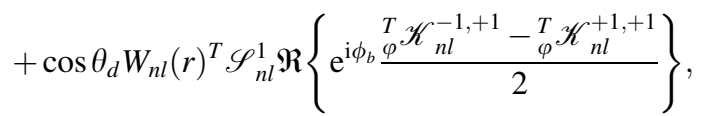

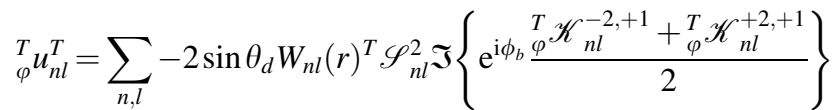

$$
\begin{aligned}
& +\cos 2 \theta_{d} W_{n l}(r)^{T} \mathscr{S}_{n l}^{1} \mathfrak{\Im}\left\{\mathrm{e}^{\mathrm{i} \phi_{b}{ }^{T} \frac{\mathscr{K}_{n l}^{-1,+1}-{ }_{\varphi}^{T} \mathscr{K}_{n l}^{+1,+1}}{2}}\right\},
\end{aligned}
$$

where ${ }^{T} \mathscr{S}_{n l}^{1},{ }^{T} \mathscr{S}_{n l}^{2}$ and ${ }_{\gamma}^{K} \mathscr{K}_{n l}^{\alpha, \beta}$ are given in "Appendix." $\phi_{b}$ is the back-azimuth angle (see Fig. 1). Other parts of displacement are shown in "Appendix." Note that as the length of the fault goes to zero, the formulae for the displacements due to a finite source become exactly the same as the point source case (Yang et al. 2010).

\section{Numerical results}

To show the impact of the transverse propagation of rupture on seismograms, we continue to make following simplifications:

(i) The fault is vertical and the slip only has strike-slip component, i.e., $\theta_{d}=\frac{\pi}{2}$,

(ii) The rupture velocity is a pulse propagating along the fault with a constant velocity, which is 
Table 1 Parameters in the numerical experiment

\begin{tabular}{ll}
\hline Source depth & $15 \mathrm{~km}$ \\
Seismic moment & $1.06 \times 10^{27}$ dyne cm \\
Earth structure & Continental PREM model \\
Normal mode & $n \leq 300, l \leq 2000$ \\
Filter & $1-100 \mathrm{mHz}$ \\
\hline
\end{tabular}

$\Delta \dot{s}_{\varphi}(\varphi, \tau)=\Delta \dot{s}_{0} \delta\left(\tau-\frac{\varphi}{v_{f}}\right)$,

where the rupture velocity $v_{f}$ is constant, and with unit $\mathrm{rad} / \mathrm{s}$.

(iii) The receiver coplanar to the fault plane.

We calculate the synthetic seismograms in different scenarios (different rupture length and rupture velocity, rupture toward and away from the receiver, respectively), show the features of seismograms of finite fault and compare them with the seismograms of point source, which is exactly the same as the result of MINEOS. Table 1 shows the source and structure parameters we use for our numerical calculation. Note that the source parameters are chose according to the study of the 1992 Landers earthquake (Wald and Heaton 1994).
Table 2 shows the four cases for our numerical experiment. The rupture velocity is represented in $\mathrm{deg} / \mathrm{s}$. Note that the rupture length is about $70 \mathrm{~km}$ and the rupture velocity is approximately $0.024 \mathrm{deg} / \mathrm{s}$ for the 1992 Landers earthquake (Wald and Heaton 1994).

Our numerical results are shown in Figs. 2, 3, 4 and 5. We can observe that the seismograms for finite sources have smaller amplitudes than those for point sources. The amplitudes are in general smaller for sources with larger rupture lengths, lower rupture velocities and when the receivers are on the back of the rupture. Moreover, the zero-amplitude "knots" can be observed in the surface waves excited by finite sources. In the next section, we give a very intuitive explanation for these phenomenons and attribute them to the interference of the waves emitted by different parts of the fault.

\section{An intuitive explanation for the numerical results}

In this section, we attempt to explain the features in the seismograms of finite faults shown in our numerical results with a simple and intuitive model. Aki and Richards (2002) used the unidirectional rectangular fault model (Haskell
Table 2 Parameters for different cases in the numerical experiment

\begin{tabular}{llll}
\hline & Rupture length $(\mathrm{km})$ & Rupture velocity $(\mathrm{deg} / \mathrm{s})$ & Direction \\
\hline Case 1 & $5,10,20,70$ & 0.024 & Front \\
Case 2 & $5,10,20,70$ & 0.024 & Back \\
Case 3 & 70 & $0.028,0.024,0.022,0.020$ & Front \\
Case 4 & 70 & $0.028,0.024,0.022,0.020$ & Back \\
\hline
\end{tabular}

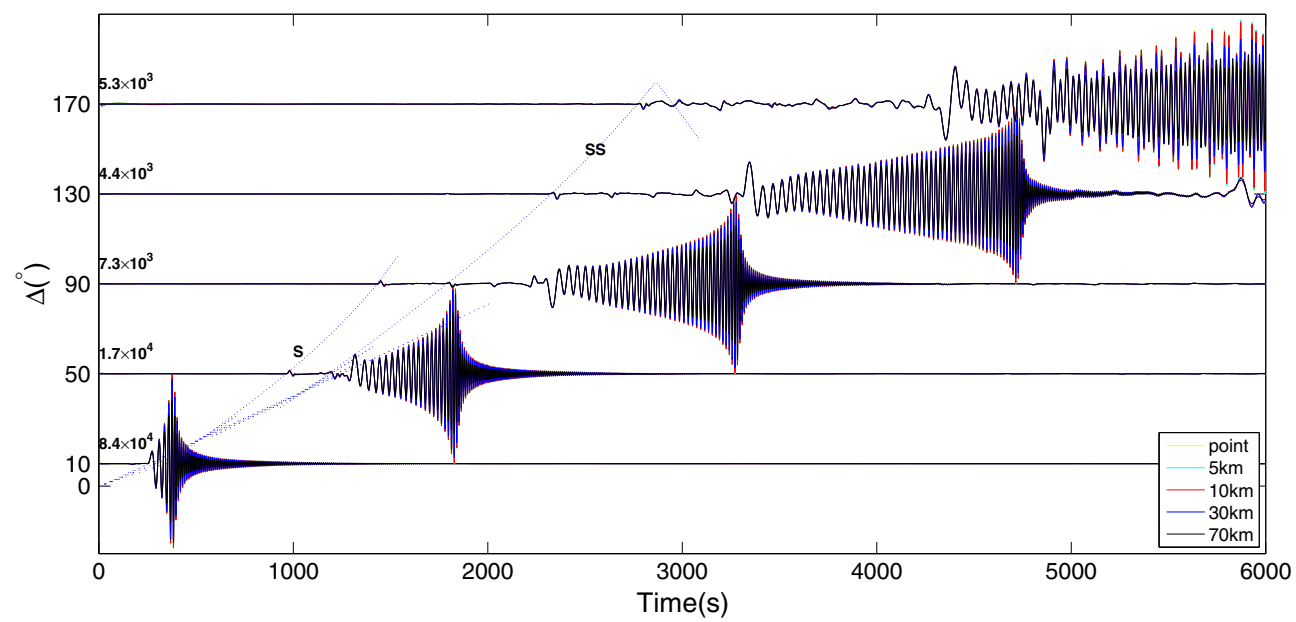

Fig. 2 Transverse component of the synthetic seismograms in front of the rupture for sources with the same rupture velocity, the same seismic moment but different rupture length. The dotted line is travel time curves of S and SS phase generated by TauP Toolkit. The rupture velocity is $0.024 \mathrm{deg} / \mathrm{s}$, and the rupture length is $5 \mathrm{~km}$ (light blue), $10 \mathrm{~km}$ (red), $30 \mathrm{~km}$ (dark blue) and $70 \mathrm{~km}$ (black). The yellow line is the synthetic seismogram for point source. The waveforms are normalized according to the maximum amplitude in the point source seismogram with the same epicentral distance. The number on the left is the maximum amplitude in the point source seismogram. Note that we plot the seismograms throughout this paper if not stated otherwise. We can observe from this figure that the synthetic seismograms in front of the rupture for every rupture length are all very similar to those of the point sources 


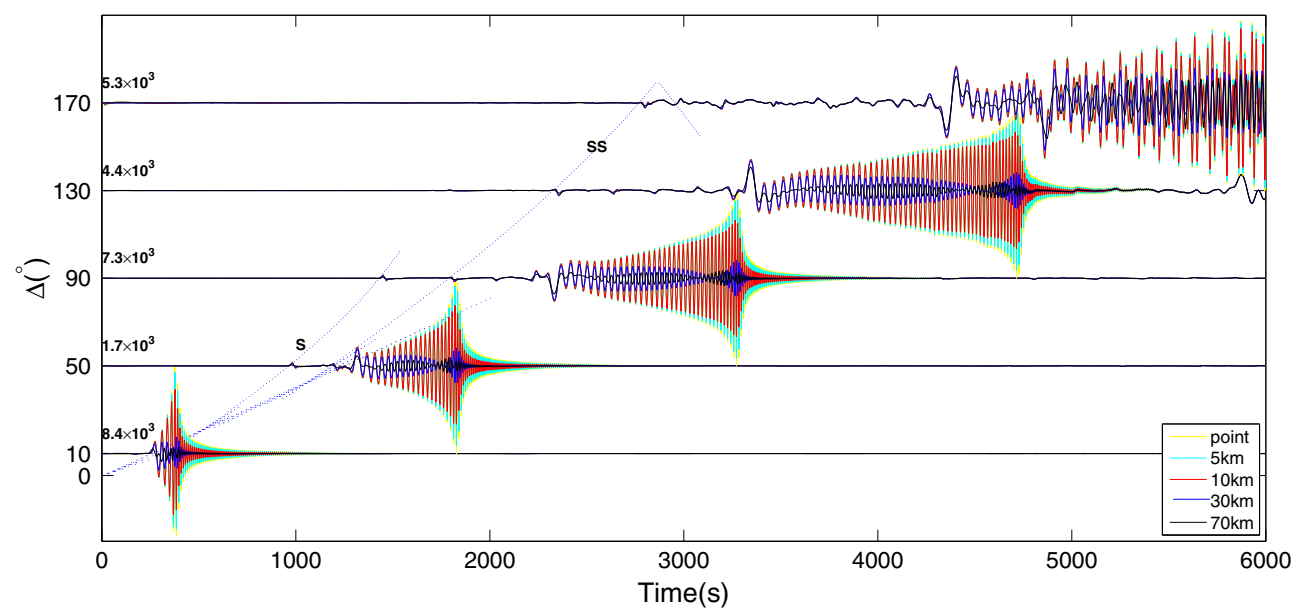

Fig. 3 Transverse component of the synthetic seismograms on the back of the rupture for sources with the same rupture velocity, the same seismic moment but different rupture length. The dotted line is travel time curves of S and SS phase generated by TauP Toolkit. The rupture velocity is $0.024 \mathrm{deg} / \mathrm{s}$, and the rupture length is $5 \mathrm{~km}$ (light blue), $10 \mathrm{~km}$ (red), $30 \mathrm{~km}$ (dark blue) and $70 \mathrm{~km}$ (black). The yellow line is the synthetic seismogram for point source. Note that the synthetic seismograms on the back of the rupture have smaller amplitudes than those of point sources. Moreover, for large rupture length, zero-amplitude "knots" can be observed

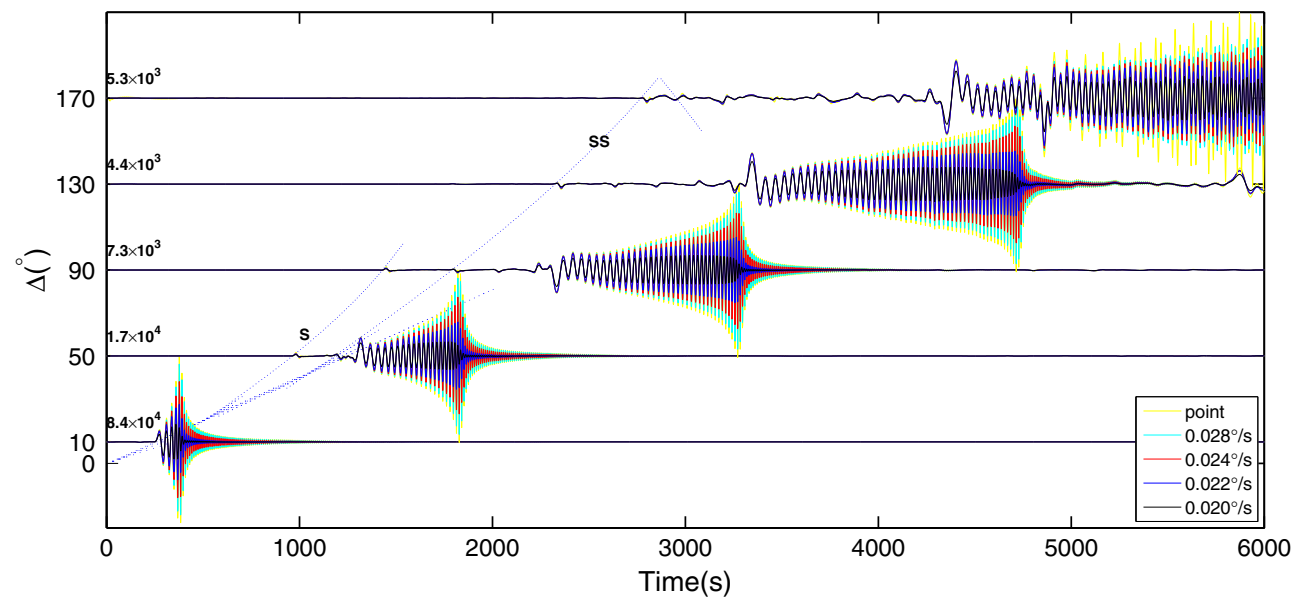

Fig. 4 Transverse component of the synthetic seismograms in front of the rupture for sources with the same rupture length, the same seismic moment but different rupture velocity. The dotted line is travel time curves of S and SS phase generated by TauP Toolkit. The rupture length is $70 \mathrm{~km}$, and the rupture velocity is $0.028 \mathrm{deg} / \mathrm{s}$ (light blue), $0.024 \mathrm{deg} / \mathrm{s}$ (red), $0.022 \mathrm{deg} / \mathrm{s}$ (dark blue) and $0.020 \mathrm{deg} / \mathrm{s}$ (black). The yellow line is the synthetic seismogram for point source. Note that the seismograms of sources that propagate slowly have small amplitudes, especially for the high-frequency component

model) to explain the spectral features of the surface waves. Using this idea, we can qualitatively explain the variation of amplitudes of the Love waves due to the rupture length and velocity of the finite faults in time domain. Suppose that we have a 1-D plane dispersive wave excited by a point source

$f(x, t)=\frac{1}{\sqrt{2 \pi}} \int_{-\infty}^{+\infty} F(\omega) \mathrm{e}^{\mathrm{i} \omega\left(\frac{x}{v(\omega)}-t\right)} \mathrm{d} \omega$,

in which $F(\omega)$ denotes the spectrum, and $v(\omega)$ is the phase velocity. For the finite source case, the wave field can be represented as the integral over the source $f(x, t)=\frac{1}{\sqrt{2 \pi}} \int_{-l}^{l} \int_{-\infty}^{+\infty} \frac{F(\omega)}{2 l} \mathrm{e}^{i \omega\left(\frac{x-x^{\prime}}{v(\omega)}-\left(t-t^{\prime}\left(x^{\prime}\right)\right)\right)} \mathrm{d} \omega \mathrm{d} x^{\prime}$

where $t^{\prime}$ is the time that the rupture arrives at $x^{\prime}$. If we further assume that the rupture propagates at a constant velocity $v_{r}$, then Eq. (16) becomes

$f(x, t)=\frac{1}{\sqrt{2 \pi}} \int_{-\infty}^{+\infty} F(\omega) \gamma(\omega) \mathrm{e}^{i \omega\left(\frac{x}{v(\omega)}-t\right)} \mathrm{d} \omega$,

where 


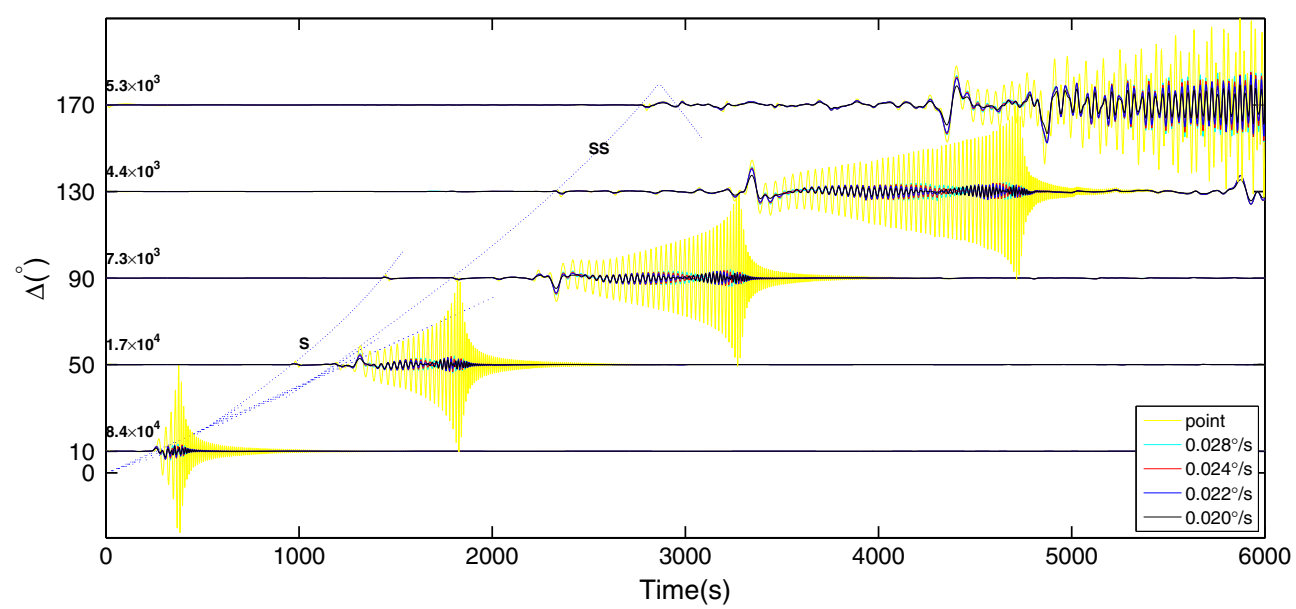

Fig. 5 Transverse component of the synthetic seismograms in front of the rupture for sources with the same rupture length, the same seismic moment but different rupture velocity. The dotted line is travel time curves of S and SS phase generated by TauP Toolkit. The rupture length is $70 \mathrm{~km}$, and the rupture velocity is $0.028 \mathrm{deg} / \mathrm{s}$ (light blue), $0.024 \mathrm{deg} / \mathrm{s}$ (red), $0.022 \mathrm{deg} / \mathrm{s}$ (dark blue) and $0.020 \mathrm{deg} / \mathrm{s}$ (black). The yellow line is the synthetic seismogram for point source. Note that the synthetic seismograms on the back of the rupture have smaller amplitudes than those of point sources. Moreover, for large rupture length, zero-amplitude "knots" can be observed

$\gamma(\omega)=\operatorname{sinc}\left[\omega l\left(\frac{1}{v_{r}}-\frac{1}{v(\omega)}\right)\right]=\operatorname{sinc}\left[\pi\left(n_{t}-n_{l}\right)\right]$.

$n_{t}$ and $n_{l}$ are ratios of rupture time over period of wave and rupture length over wavelength,

$n_{t}=\frac{T_{r}}{T}, \quad n_{l}=\frac{2 l}{\lambda}$.

Here we define $v_{r}$ and $T_{r}$ to be negative if the rupture propagates away from the receiver. The "sinc" function in Eq. (17) is

$\operatorname{sinc}(x)=\frac{\sin x}{x}$,

the graph of which is shown in Fig. 6 .

Note that the only difference between the wave field excited by point source Eq. (15) and that by finite source Eq. (17) is the existence of $\gamma$, which is the amplification coefficient due to the effect of finite source. Then, we can discuss the amplitude of wave in frequency domain.

Table 3 shows the value of $n_{t}-n_{l}$ in different scenarios. The dispersion relation for PREM model is given by Widmer-Schnidrig and Laske (2009). According to our model, if $\left|n_{t}-n_{l}\right| \approx 0$ then $\gamma \approx 1$, the wave emitted by all parts of the source arrives approximately at the same time, the amplitude is almost as large in the wave field produced by finite source as by point source; if $\left|n_{t}-n_{l}\right| \ll 1$, then $\gamma \approx 0$, which means that the amplitude is greatly diminished due to the destructive interference of the wave emitted by different parts of the source. The idea here is essentially the same as in Vallée and Dunham (2012). Moreover, if $n_{t}-n_{l}$ is a nonzero integer, $\gamma=0$ which indicates the position of the zero-amplitude "knot." From Table 3, we can see that

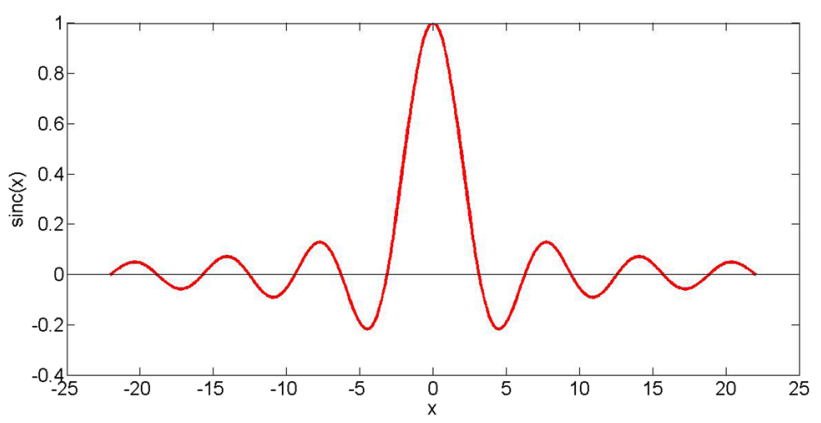

Fig. 6 Graph of the "sinc" function. $\operatorname{sinc}(0)=1$ is the maximum of the function, and the value rapidly decays away from 0 . Moreover, $\operatorname{sinc}(n \pi)=0$ for $n$ is nonzero integer

(i) For the same rupture length and velocity, the finiteness of the fault impacts more on high-frequency component than on low-frequency component;

(ii) For the same frequency, the waves produced by longer faults have smaller amplitudes than those produced by shorter faults;

(iii) The waves have larger amplitude in front of the rupture than in the back and have more zeroamplitude knots,

which correspond exactly to what we observe in the numerical results. Moreover, note in Table 3 that when rupture length $111.2 \mathrm{~km}$, rupture velocity $0.020 \mathrm{deg} / \mathrm{s}$ and frequency $f=50 \mathrm{mHz}$, the $n_{t}-n_{l}$ value is very close to 1 , which predicts the zero-amplitude knot. Actually, we can observe the knot in the synthetic seismogram exactly correspond to the $50 \mathrm{mHz}$ group velocity (Fig. 7). 
Table 3 Values of $n_{t}-n_{l}$ for some combinations of rupture length, rupture velocity and frequency

\begin{tabular}{lcrrrrr}
\hline Rupture length $(\mathrm{km})$ & Rupture velocity $(\mathrm{s} / \mathrm{deg})$ & $3 \mathrm{mHz}$ & $10 \mathrm{mHz}$ & $20 \mathrm{mHz}$ & $50 \mathrm{mHz}$ & $100 \mathrm{mHz}$ \\
\hline 70 & 0.024 & 0.0276 & 0.0744 & 0.1199 & 0.2120 & 0.2662 \\
70 & 0.020 & 0.0540 & 0.1626 & 0.2962 & 0.6527 & 1.1475 \\
111.2 & 0.020 & 0.0858 & 0.2583 & 0.4705 & 1.0368 & 1.8229 \\
10 & 0.020 & 0.0077 & 0.0232 & 0.0423 & 0.0932 & 0.1639 \\
70 & -0.024 & -0.1084 & -0.3788 & -0.7866 & -2.0541 & -4.2662 \\
70 & -0.020 & -0.1348 & -0.4669 & -0.9628 & -2.4948 & -5.1475 \\
\hline
\end{tabular}

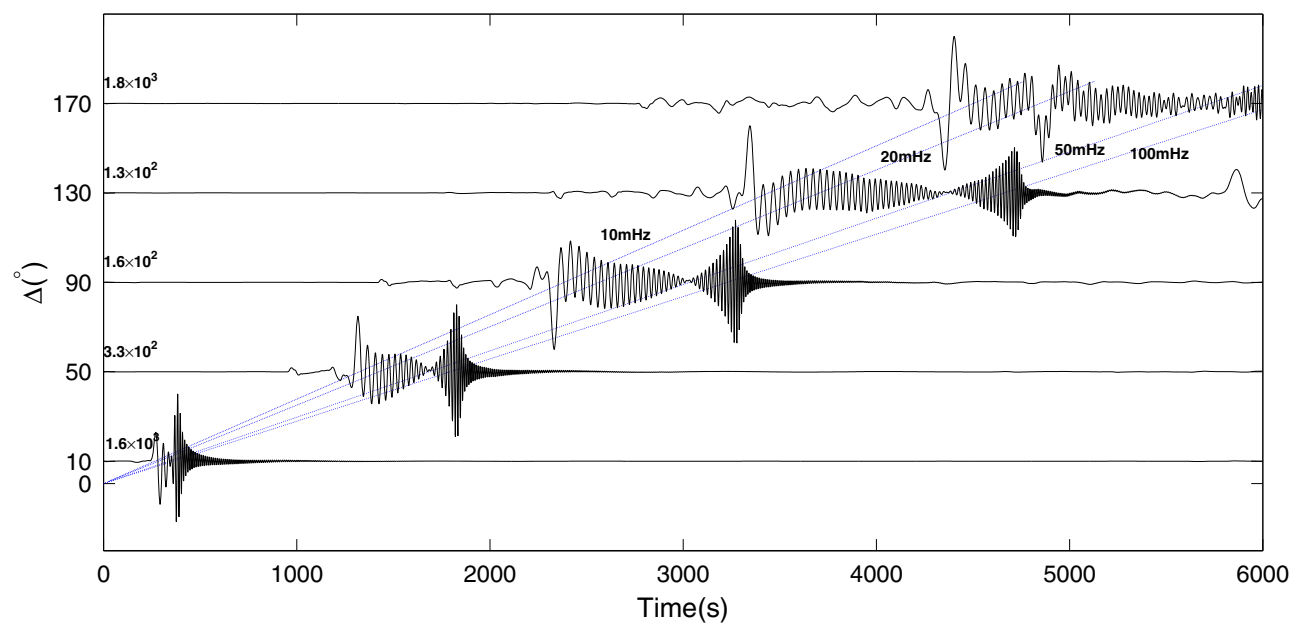

Fig. 7 Zero-amplitude "knots" in synthetic seismograms. Note that they exist near the position that correspond to the group velocity of $50 \mathrm{mHz}$ Love wave

\section{Conclusion}

In this paper, we expand the normal-mode method to the finite source case. Instead of using point source summation, we directly calculate the excitation of each mode by the finite fault and add together to obtain the synthetic seismogram. We derive the solution of displacement produced by a "line source" and carry out numerical experiments and discuss the impact of rupture length and rupture velocity on the wave form of synthetic seismogram, especially the wave form of Love wave. We observe that

(i) The amplitude is smaller in the seismogram of finite fault than that of point source with the same seismic moment;

(ii) The finiteness of fault has more significant impact on high-frequency component than on low-frequency component;

(iii) The amplitude in the seismogram of finite fault is diminished more for the receiver that in the back of the rupture;

(iv) Zero-amplitude "knots" exist, and there are more "knots" in the back of the rupture.
Moreover, we use the Haskell model and the interference of waves emitted by different parts of the fault to provide a very intuitive explanations for all these phenomenons. Although this model is too simple to explain all the features in different cases accurately and qualitatively, it describes the basic characters of the seismogram of finite faults.

However, there are still some problems remain to be dealt with. The first one is the inclusion of radial propagation of the rupture. In this paper, we treat the fault as the "line source," which is far from enough. The line source model can only capture the effect of rupture propagation in the transverse direction. Although this is a step forward compared with the point source model, it still cannot take into account all the features on the fault. The second one is the efficiency of the method. The finiteness of the source causes the unavailability of the additional theorem of GSH, which means that the summation over $m$ needs to be numerically calculated. Such summation is quite inefficient numerically. These two problems must be solved in order for this method to be usable in the calculation of synthetic seismograms.

Acknowledgements This work was supported by the National Natural Science Foundation of China (Grant No. 41674050) and MOST grant (2012CB417301). We thank the two anonymous reviewers for 
their constructive comments and suggestions which are crucial to the improvement of our manuscript.

Open Access This article is distributed under the terms of the Creative Commons Attribution 4.0 International License (http://crea tivecommons.org/licenses/by/4.0/), which permits unrestricted use, distribution, and reproduction in any medium, provided you give appropriate credit to the original author(s) and the source, provide a link to the Creative Commons license, and indicate if changes were made.

\section{Appendix: Formulae for the synthetic seismograms of finite faults}

The displacement triggered by the finite fault is

$$
\begin{aligned}
& { }_{\xi}^{S} u^{Z}=\sum_{n, l} \sin 2 \theta_{d} U_{n l}(r)\left({ }^{S} \mathscr{S}_{n l \xi}^{0 S} \mathscr{K}_{n l}^{0,0}-{ }^{S} \mathscr{S}_{n l}^{2} \mathfrak{R}\left\{{ }_{\xi}^{S} \mathscr{K}_{n l}^{-2,0}\right\}\right) \\
& \cos 2 \theta_{d} U_{n l}(r)^{S} \mathscr{S}_{n l}^{2} \mathfrak{R}\left\{\begin{array}{l}
S \\
\xi
\end{array} \mathscr{K}_{n l}^{-1,0}\right\}, \\
& { }_{\xi}^{S} u^{R}=\sum_{n, l} \sin 2 \theta_{d} V_{n l}(r)\left({ }^{s} \mathscr{S}_{n l}^{0} \mathfrak{R}\left\{\mathrm{e}^{i \phi_{b} S}{ }_{\xi} \mathscr{K}_{n l}^{0,+1}\right\}\right. \\
& \left.-S_{\mathscr{S}_{n l}^{2} \mathfrak{R}}\left\{\mathrm{e}^{i \phi_{b}} \frac{{ }_{\xi}^{S} \mathscr{K}_{n l}^{-2,+1}+{ }_{\xi}^{S} \mathscr{K}_{n l}^{+2,+1}}{2}\right\}\right) \\
& +\cos 2 \theta_{d} V_{n l}(r)^{S} \mathscr{S}_{n l}^{1} \mathfrak{R}\left\{\mathrm{e}^{i \phi_{b}} \frac{{ }_{\xi}^{S} \mathscr{K}_{n l}^{-1,+1}-{ }_{\xi}^{S} \mathscr{K}_{n l}^{+1,+1}}{2}\right\}, \\
& { }_{\xi}^{S} u^{T}=\sum_{n, l}-\sin 2 \theta_{d} V_{n l}(r)\left({ }^{S} \mathscr{S}_{n l}^{0} \mathfrak{J}\left\{\mathrm{e}^{i \phi_{b} S}{ }_{\xi} \mathscr{K}_{n l}^{0,+1}\right\}\right.
\end{aligned}
$$

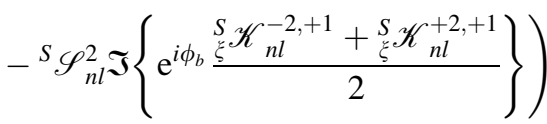

$$
\begin{aligned}
& -\cos 2 \theta_{d} V_{n l}(r)^{S} \mathscr{S}_{n l}^{1} \mathfrak{J}\left\{\mathrm{e}^{i \phi_{b}} \frac{{ }_{\xi}^{S} \mathscr{K}_{n l}^{-1,+1}-{ }_{\xi}^{S} \mathscr{K}_{n l}^{+1,+1}}{2}\right\},
\end{aligned}
$$

${ }_{\xi}^{T} u^{Z}=0$,

$$
\begin{aligned}
{ }_{\xi}^{T} u^{R}= & \sum_{n, l} \sin 2 \theta_{d} W_{n l}(r)^{T} \mathscr{S}_{n l}^{2} \mathfrak{R}\left\{\mathrm{e}^{\left.i \phi_{b} \frac{{ }_{\xi}^{T} \mathscr{K}_{n l}^{-2,+1}-{ }_{\xi}^{T} \mathscr{K}_{n l}^{+2,+1}}{2}\right\}}\right. \\
& -\cos 2 \theta_{d} W_{n l}(r)^{T} \mathscr{S}_{n l}^{1} \mathfrak{R}\left\{\mathrm{e}^{\left.i \phi_{b} \frac{{ }_{\xi} \mathscr{K}_{n l}^{-1,+1}+{ }_{\xi}^{T} \mathscr{K}_{n l}^{+1,+1}}{2}\right\},}\right. \\
{ }_{\xi}^{T} u^{T}= & \sum_{n, l}-\sin 2 \theta_{d} W_{n l}(r)^{T} \mathscr{S}_{n l}^{2} \mathfrak{J}\left\{\mathrm{e}^{\left.i \phi_{b} \frac{{ }_{\xi}^{T} \mathscr{K}_{n l}^{-2,+1}-{ }_{\xi}^{T} \mathscr{K}_{n l}^{+2,+1}}{2}\right\}}\right. \\
& +\cos 2 \theta_{d} W_{n l}(r)^{T} \mathscr{S}_{n l}^{1} \mathfrak{T}\left\{\mathrm{e}^{\left.i \phi_{b} \frac{{ }_{\xi}^{T} \mathscr{K}_{n l}^{-1,+1}+{ }_{\xi}^{T} \mathscr{K}_{n l}^{+1,+1}}{2}\right\},}\right.
\end{aligned}
$$

$$
\begin{gathered}
\left.{ }_{\varphi}^{S} u^{Z}=\sum_{n, l} 2 \sin \theta_{d} U_{n l}(r)^{S} \mathscr{S}_{n l}^{2} \mathfrak{I}\left\{\begin{array}{l}
S \\
\varphi
\end{array} \mathscr{K}_{n l}^{-2,0}\right\}\right\} \\
-\cos \theta_{d} U_{n l}\left(r_{R}\right)^{S} \mathscr{S}_{n l}^{2} \mathfrak{J}\left\{{ }_{\xi}^{S} \mathscr{K}_{n l}^{-1,0}\right\},
\end{gathered}
$$$$
{ }_{\varphi}^{S} u^{R}=\sum_{n, l} 2 \sin \theta_{d} V_{n l}(r)^{S} \mathscr{S}_{n l}^{2} \mathfrak{J}\left\{\mathrm{e}^{i \phi_{b}} \frac{{ }_{\varphi} \mathscr{K}_{n l}^{-2,+1}-{ }_{\varphi}^{S} \mathscr{K}_{n l}^{+2,+1}}{2}\right\}
$$$$
-\cos \theta_{d} V_{n l}(r)^{S} \mathscr{S}_{n l}^{1} \mathfrak{J}\left\{\mathrm{e}^{i \phi_{b} \frac{S \varphi \mathscr{K}_{n l}^{-1,+1}+{ }_{\varphi}^{S} \mathscr{K}_{n l}^{+1,+1}}{2}}\right\},
$$

$$
\begin{aligned}
{ }_{\varphi}^{S} u^{R}= & \sum_{n, l} 2 \sin \theta_{d} V_{n l}(r)^{S} \mathscr{S}_{n l}^{2} \mathfrak{R}\left\{\mathrm{e}^{\left.i \phi_{b} \frac{{ }_{\varphi} \mathscr{K}_{n l}^{-2,+1}-{ }_{\varphi}^{S} \mathscr{K}_{n l}^{+2,+1}}{2}\right\}}\right. \\
& -\cos \theta_{d} V_{n l}(r)^{S} \mathscr{S}_{n l}^{1} \mathfrak{R}\left\{\mathrm{e}^{\left.i \phi_{b} \frac{{ }_{\varphi} \mathscr{K}_{n l}^{-1,+1}+{ }_{\varphi} \mathscr{K}_{n l}^{+1,+1}}{2}\right\},}\right.
\end{aligned}
$$

$$
\begin{aligned}
& { }_{\varphi}^{T} u^{Z}=0 \text {, } \\
& { }_{\varphi}^{T} u^{R}=\sum_{n, l}-2 \sin \theta_{d} W_{n l}(r)^{T} \mathscr{S}_{n l}^{2} \mathfrak{R}\left\{\mathrm{e}^{i \phi_{b}} \frac{{ }_{\varphi}^{T} \mathscr{K}_{n l}^{-2,+1}+{ }_{\varphi}^{T} \mathscr{K}_{n l}^{+2,+1}}{2}\right\}
\end{aligned}
$$

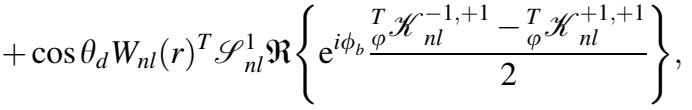

$$
\begin{aligned}
& { }_{\varphi}^{T} u^{T}=\sum_{n, l}-2 \sin \theta_{d} W_{n l}(r)^{T} \mathscr{S}_{n l}^{2} \mathfrak{J}\left\{\mathrm{e}^{i \phi_{b} \varphi^{T} \mathscr{K}_{n l}^{-2,+1}+{ }_{\varphi}^{T} \mathscr{K}_{n l}^{+2,+1}} \frac{2}{2}\right\} \\
& +\cos 2 \theta_{d} W_{n l}(r)^{T} \mathscr{S}_{n l}^{1} \mathfrak{J}\left\{\mathrm{e}^{i \phi_{b}} \frac{{ }_{\varphi}^{T} \mathscr{K}_{n l}^{-1,+1}-{ }_{\varphi}^{T} \mathscr{K}_{n l}^{+1,+1}}{2}\right\},
\end{aligned}
$$

where

$$
\begin{aligned}
& s_{\mathscr{S}_{n l}^{0}}=\dot{U}_{n l}\left(r_{f}\right)+\frac{1}{r_{f}}\left(\frac{\sqrt{l(l+1)}}{2} V_{n l}\left(r_{f}\right)-U_{n l}\left(r_{f}\right)\right), \\
& s_{\mathscr{S}_{n l}^{1}}=\dot{V}_{n l}\left(r_{f}\right)+\frac{1}{r_{f}}\left(\sqrt{l(l+1)} U_{n l}\left(r_{f}\right)-V_{n l}\left(r_{f}\right)\right), \\
& s_{\mathscr{S}_{n l}^{2}}=\frac{\sqrt{(l-1)(l+2)}}{2 r_{f}} V_{n l}\left(r_{f}\right), \\
& { }^{T} \mathscr{S}_{n l}^{1}=\dot{W}_{n l}\left(r_{f}\right)-\frac{W_{n l}\left(r_{f}\right)}{r_{f}}, \quad{ }^{T} \mathscr{S}_{n l}^{2}=\frac{\sqrt{(l-1)(l+2)}}{2 r_{f}} W_{n l}\left(r_{f}\right),
\end{aligned}
$$

and 


$$
\begin{aligned}
& { }_{\gamma}^{K} \mathscr{K}_{n l}^{\alpha, \beta}(\boldsymbol{x}, t)=\frac{2 l+1}{4 \pi} \mu w r_{f} \sin \theta_{d} \\
& \times \sum_{m=-l}^{+l}\left(P_{l m}^{\alpha}\left(\cos \theta_{d}\right) P_{l m}^{\beta}(\cos \theta) \mathrm{e}^{i m \phi} \int_{\varphi_{1}}^{\varphi_{2}} \int_{0}^{T_{f}} \Delta \dot{s}_{\gamma}(\varphi, \tau) \mathrm{e}^{-i m \varphi}\right. \\
& \left.\quad \times \frac{1-\mathrm{e}^{-{ }^{K} \sigma_{n l}(t-\tau)} \cos ^{K} \omega_{n l}(t-\tau)}{K^{K} \omega_{n l}^{2}} \mathrm{~d} \tau \mathrm{d} \varphi\right)
\end{aligned}
$$

\section{References}

Aki K, Richards PG (2002) Quantitative seismology, 2nd edn. University Science Books, Sausalito, pp 491-536

Ben-Menahem A, Singh SJ (1987) Supershear accelerations and Mach-waves from a rupturing front-I. Theoretical model and implications. J Phys Earth 35:347-365

Bielak J, Loukakis K, Hisada Y, Yoshimura C (2003) Domain reduction method for three-dimensional earthquake modeling in localized regions, part I: theory. Bull Seismol Soc Am 93:817-824

Boore DM (1972) Finite-difference methods for seismic wave propagation in heterogeneous materials. In: Bolt BA (ed) Methods in computational physics. Academic Press, NY, pp 1-37

Bouchon M, Aki K (1977) Discrete wave-number representation of seismic-source wave fields. Bull Seismol Soc Am 67:259-277

Bouchon M (1980a) The motion of the ground during and earthquake 1. The case of a strike slip fault. J Geophys Res 85:356-366

Bouchon M (1980b) The motion of the ground during and earthquake 2. The case of a dip slip fault. J Geophys Res 85:367-375

Chapman CH (1978) A new method for computing synthetic seismograms. Geophys J R Astron Soc 54:481-518

Dahlen FA, Tromp J (1998) Theoretical global seismology. Princeton University Press, Princeton, pp 363-404

Gilbert F (1971) Excitation of normal modes of earth by earthquake sources. Geophys J R Astron Soc 22:223-226

Gilbert F, Helmberger DV (1972) Generalized ray theory for a layered sphere. Geophys J R Astron Soc 27:57-80

Israel M, Kovach RL (1977) Near-field motions from a propagating strike-slip fault in an elastic Half-space. Bull Seismol Soc Am 67:977-994

Kennett BLN, Kerry NJ (1979) Seismic wave in a stratified half space. Geophys J Int 57:557-583

Komatitsch D, Tromp J (1999) Introduction to the spectral element method for three-dimensional seismic wave propagation. Geophys J Int 139:806-822
Komatitsch D, Tromp J (2002) Spectral-element simulations of global seismic wave propagation-II. Three-dimensional models, oceans, rotation and self-gravitation. Geophys J Int 150:303-318

Luco JE, Apsel RJ (1983) On the Green's functions for a layered halfspace. Part I. Bull Seismol Soc Am 73:909-929

Phinney RA, Burridge R (1973) Representation of elastic-gravitational excitation of a spherical earth model by generalized spherical harmonics. Geophys J R Astron Soc 34:451-487

Saikia CK, Helmberger DV (1997) Approximation of rupture directivity in regional phases using upgoing and downgoing wave fields. Bull Seismol Soc Am 87:987-998

Singh SJ, Ben-Menahem A (1969a) Eigenvibrations of the earth excited by finite dislocations-I. Toroidal oscillations. Geophys J R Astron Soc 17:151-177

Singh SJ, Ben-Menahem A (1969b) Eigenvibrations of the earth excited by finite dislocations-II. Spheroidal oscillations. Geophys J R Astron Soc 17:333-350

Song XJ, Helmberger DV (1996) Source estimation of finite faults from broadband regional networks. Bull Seismol Soc Am 86:797-804

Stump BW, Johnson LR (1982) Higher-degree moment tensors-the importance of source finiteness and rupture propagation on seismograms. Geophys J R Astron Soc 69:721-743

Takeuchi H, Saito M (1972) Seismic surface waves. In: Bruce AB (ed) Seismology: surface waves and earth oscillations, volume 11 of methods in computational physics. Academic Press, New York, pp 217-295

Tanimoto T (1984) A simple derivation of the formula to calculate synthetic long-period seismograms in a heterogeneous earth by normal mode summation. Geophys J R Astron Soc 77:275-278

Vallée M, Dunham EM (2012) Observation of far-field Mach waves generated by the 2001 Kokoxili supershear earthquake. Geophys Res Lett 39:L05311

Wald DJ, Heaton TH (1994) Spatial and temporal distribution of slip for the 1992 Landers, California, earthquake. Bull Seismol Soc Am 84:668-691

Widmer-Schnidrig R, Laske G (2009) Theory and observationsnormal modes and surface wave measurements. In: Dziewonski AM, Romanowicz BZ (eds) Seismology and structure of the earth: treatise on geophysics. Elsevier, Amsterdam, pp 67-125

Woodhouse JH (1988) The calculation of the eigenfrequencies and eigenfunctions of the free oscillations of the Earth and the Sun. In: Doorbos DJ (ed) Seismological algorithms. Academic Press, San Diago, pp 321-370

Yang H-Y, Zhao L, Hung S-H (2010) Synthetic seismograms by normal-mode summation: a new derivation and numerical examples. Geophys J Int 183:1613-1632 